\title{
MAP Optimum Receiver Mitigating Correlated Impulsive Noise
}

\author{
Fabien Sacuto \\ McGill University \\ Montreal, Canada \\ Email: \\ fabien.sacuto@mail.mcgill.ca
}

\author{
Gaetan Ndo \\ Électricité de France \\ France \\ Email: \\ gaetan.ndo@gmail.com
}

\author{
Fabrice Labeau \\ McGill University \\ Montreal, Canada \\ Email: \\ fabrice.labeau@mcgill.ca
}

\author{
Basile.L Agba \\ IREQ \\ Varennes, Canada \\ Email: \\ agba.basile@ireq.ca
}

\begin{abstract}
Power substations generate a significant "bursty impulse noise" that might interfere with wireless technologies working in the vicinity of power equipment. Existing wireless systems are not designed for such an environment; we propose a Maximum a Posteriori (MAP) receiver designed with Markov-Gaussian models in order to mitigate the impact of impulsive noise in substations. We study and compare different noise models implemented in the receiver and we discuss the performance of the receiver based on the characteristics of the impulsive noise. Our proposed model can be used by a MAP receiver to offer optimum performances from low signal to noise ratio (SNR). When the communication is disturbed by impulsive noise measured in the field, the MAP receiver still offers better performance than when using other models, but mainly at higher SNR.
\end{abstract}

\section{INTRODUCTION}

IEC 61850 standard integrates Substation Automation (SA) for Smart Grid [1], which requires installing Intelligent Electrical Devices (IED) within substations. Wireless technology remains a serious candidate for such an application, since it allows an easy installation of sensors and saves expensive retrofitting in substations. However, the power equipment and electrical switching operations generate a significant impulsive noise that might disturb wireless communications. Impulsive noise can be represented by a process switching between a background noise and impulses. In a noise modeling framework, the background noise is usually represented by a Gaussian distribution, while the impulses can have a different pattern, depending on the model. Wide-band measurements in substations [2] show that impulses have a damped oscillating waveform, which means that the samples are correlated. In [3], we have seen that the power spectrum of the impulses is significant enough to be detected by wireless receivers, which motivates the design of new receivers that use the memory of impulsive noise models to enhance the communication.

The receiver can use the correlation between the samples with an implemented statistical model in order to estimate the transmitted bits; hence, models with memory would improve the detector performances on the receiver side. In our previous works [4], [5], we have presented our impulsive noise model that is capable of replicating the damped oscillation waveform within the impulses. In the present paper, we propose to implement a new less complex model, in terms of the number of noise states, into a MAP receiver. Finally, we observe the performances at the receiver output when the channel is disturbed by substation impulsive noise.

We present in Section II some impulsive noise models such as Bernoulli-Gaussian with memory and a new version of our proposed Partitioned Markov chain. In Section III, we present the design of a MAP optimum receiver for the Markov-Gaussian channel and we explain how the detector calculates the output probabilities that are used for the decoding. In Section IV, we present the simulation conditions and we show the BER results for a communication between a transmitter and a receiver that is impaired by both synthetic and measured impulsive noise.

\section{IMPULSIVE NOISE MODELS}

Among different models representing impulsive noise, two different types emerge: memoryless models and models with correlated samples. Middleton's class-A model (MCA) is used a lot in communications under impulsive noise and optimum receivers using MCA offer satisfying performances for average and strong impulsive noise [6]. In [6] a simplification of the model decreases the complexity in terms of calculations by identifying the noise with two states: one for the background noise and one for the impulse. Each state is associated with a Gaussian distribution, where the 
variance of the impulse is larger than the variance of the background noise; such a simplification can be interpreted as a Bernoulli-Gaussian model.

In this work, we will consider the Bernoulli-Gaussian model with memory, which is a two-sate Markov-chain (MC2), as a classic model for "bursty" impulsive noise. We will compare the performances resulting in the use of the MC2 model with our own configuration of a Partitioned Markov chain (PMC).

\section{A. Bernoulli-Gaussian}

1) Memoryless model: The Bernoulli-Gaussian model (BG) is certainly the simplest model that can represent impulsive noise since it is a mixture of two zeromean Gaussian distributions ruled by a single Bernoulli probability [7]. The probability density function (PDF) of the amplitudes $f\left(x \mid \sigma_{0}^{2}, \sigma_{1}^{2}, p\right)$ for this model is

$$
f\left(x \mid \sigma_{0}^{2}, \sigma_{1}^{2}, p\right)=\frac{1-p}{\sqrt{2 \pi \sigma_{0}^{2}}} \exp \left(-\frac{x^{2}}{2 \sigma_{0}^{2}}\right)+\frac{p}{\sqrt{2 \pi \sigma_{1}^{2}}} \exp \left(-\frac{x^{2}}{2 \sigma_{1}^{2}}\right)
$$

In general cases, one Gaussian distribution characterizes the background noise and the other, the impulses. The BG model assumes there is only one source of impulsive noise that generates i.i.d. impulses for a one-sample duration. According to (1), the model needs only three parameters, which are the background noise variance $\sigma_{0}^{2}$, the impulses variance $\sigma_{1}^{2}$, and the Bernoulli probability $p$ to be in an impulsive state.

2) Two-states Markov chain: One implements memory to the Bernoulli-Gaussian model by associating a Gaussian distribution to each noise state of a Markov chain and by choosing appropriate transition probabilities between the states (Figure 1). The Bernoulli-Gaussian model can generate impulses with samples that follow a Gaussian distribution; the variance of the impulse remains larger than the one of the background noise. The duration of the impulse is configured with the probability to remain in the impulse state (state $I$ in Figure 1). Different methods for parameter estimation exist, such as the method of moments and the speEM algorithm. We prefer to calculate the parameters with the output of our impulse detection method presented in [4]. Our impulse detection method detects and separates the impulses from the background noise, which allows us to get information such as the impulse generation rate, the average impulse duration, the variances of both the background noise and the impulses. We work with a Maximum-Likelihood (ML) approach in the study of impulsive noise; in order to estimate the variance of the impulses and the probability to remain in the impulse state, we calculate respectively the sample variance and the average duration of the impulses. The transitions probabilities are derived from the impulse rate and the average impulse durations:

$$
\begin{gathered}
p=\frac{\text { Number of impulse events }}{\text { Number of background noise samples }} \\
q=1-\frac{1}{\text { Average number of samples per impulse }}
\end{gathered}
$$

This model is actually a particular configuration of the partitioned Markov chain [7], [8] since one state represents the background noise (state $G$ ) and the other represents the impulses (state $I$ ).

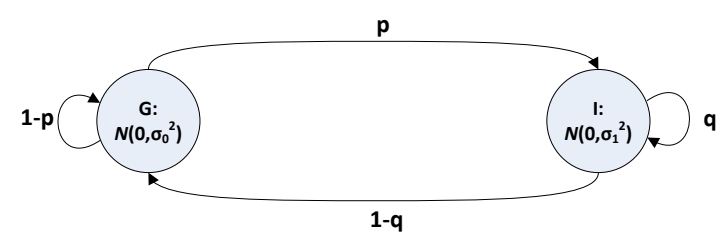

Fig. 1. Bernoulli-Gaussian model with memory.

\section{B. Partitioned Markov chain}

The partitioned Markov chain represents impulses events for Power Line Communications (PLC) [8]. In our previous works [4], we have modified the configuration of [8] to design our own impulsive noise PMC model. Using 19 states where one state represents the background noise and the 18 other states represent the impulses, we manage to generate an impulsive noise with characteristics similar to those derived from measurements in substations. The model associates a Gaussian distribution $\mathcal{N}\left(\mu_{m}, \sigma_{m}^{2}\right)$ to each state $m$, where $\mu_{m}$ and $\sigma_{m}^{2}$ are the mean and the variance respectively. Such a model provides the correlation required for a wide band representation of substation impulsive noise, since characteristics such as impulse duration, sample distribution, impulse amplitude and energy density spectrum of the impulses are better replicated than with other models. The parameters for our PMC model are estimated from measurements by using a Fuzzy C-means algorithm [5]. The proposed configuration of PMC provide a very accurate output to represent impulsive noise in substations; however, the 19 Gaussian distributions make the model very complex for parameter estimation and for an implementation in a receiver. 
TABLE I

TRANSITION MATRIX OF THE PROPOSED PARTITIONED MARKOV CHAIN WITH 6 IMPULSIVE STATES PMC-6

$$
T=\left(\begin{array}{ccccccc}
p_{00} & 0 & 0 & p_{01} & 0 & 0 & p_{01} \\
p_{10} & p_{f} & q_{10} & 0 & 0 & 0 & 0 \\
p_{10} & 0 & p_{f} & q_{10} & 0 & 0 & 0 \\
p_{10} & 0 & 0 & p_{f} & q_{10} & 0 & 0 \\
p_{10} & 0 & 0 & 0 & p_{f} & q_{10} & 0 \\
p_{10} & 0 & 0 & 0 & 0 & p_{f} & q_{10} \\
p_{10} & q_{10} & 0 & 0 & 0 & 0 & p_{f}
\end{array}\right),
$$

with $q_{10}=1-p_{f}-p_{10}$ and $p_{00}=1-2 \times p_{01}$.

For a simpler implementation of the model into a receiver, we propose to lighten the PMC model by representing the impulses with 6 states instead of 18 . We call this new version PMC6. The transition matrix of the PMC6 model is configured as indicated in Table I. The state 0 represents the background noise and the states $1,2 \ldots, 6$ represent the impulse. The transition from the background noise to the impulses is represented by the probability $p_{01}$ which corresponds to the half of the probability $p$ used in the Bernoulli-Gaussian model (Figure 1). $p_{10}$ is the probability to leave any state of the impulse and to return to the background noise state (state 0). For simplifying our model, we consider that any state of an impulse has the same probability to return to state 0 , which is equivalent to the probability $1-q$ in MC2 model (Figure 1). The probability $p_{f}$ to remain in a state of an impulse characterizes the oscillation frequency that is observed in an impulse [4]. The equation used to calculate the probability $p_{f}$ are explained in (4):

$$
p_{f}=1-6 \frac{f}{F_{s}}
$$

with $f$ the oscillation frequency in $\mathrm{MHz}$ and $F_{s}$ the sampling frequency used by the oscilloscope. Due to our measurement setup, we choose $f=700 \mathrm{MHz}$ and $F_{s}=$ $5 \mathrm{GS} / \mathrm{s}$. We provide all the parameter values in Section IV.

\section{MAP RECEIVER}

In [9], the authors acknowledge that if the noise was correlated, the proposed receiver using the Middleton class-A model would not be optimum and only a receiver considering the correlation could reach optimum performances. We propose to use a MAP receiver that is composed of three Soft-Input Soft-Output (SISO) blocks: a detector, a de-mapper and a decoder, and which produces decoded data at the bit level (Figure 2). Such a receiver is designed for communications under "bursty impulsive noise" as we can find in Power Line
Communications [10], [11]. The system consists of a communication between a transmitter and a receiver where the transmission is corrupted by correlated impulsive noise. The system uses a BPSK mapping. Before going any further, we define the following terms:

- $c_{k}$ : Transmitted symbol at time $k$

- $z_{k}$ : Noise component at time $k$

- $s_{k}$ : Noise state at instant $k$

- $K$ : Codeword length

- $R_{1}^{K}$ : Received sequence of length $K$

- $r_{k}$ : Received symbol at time $k$

- $b_{i, k}: i^{t h}$ bit of the symbol $c_{k}$

- $\hat{b}_{i, k}$ : Estimation of the transmitted bit $b_{i, k}$

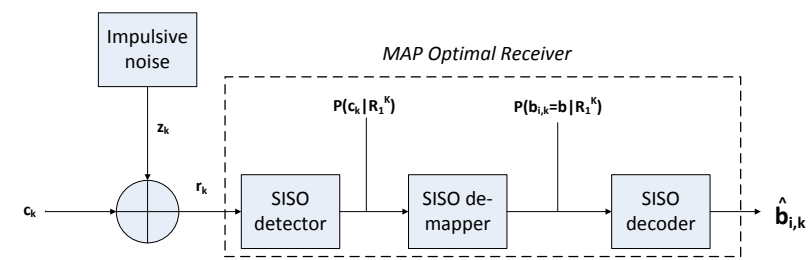

Fig. 2. MAP optimal receiver.

The system is represented by equation (5); at time slot $k$.

$$
r_{k}=c_{k}+z_{k} \quad, \quad k \in\{1,2, \ldots, K\} .
$$

The detector calculates the a-posteriori probability $p\left(c_{k} \mid R_{1}^{K}\right)$ that the symbol $c_{k}$ is emitted based on the observation of the received sequence $R_{1}^{K}$ by using the BCJR algorithm (Bahl, Cocke, Jelinek, Raviv) [12]. We present below the terms used in the algorithm :

$$
\begin{aligned}
F_{k}\left(c_{k}, s_{k}, s_{k+1}\right)= & p\left(s_{k+1} \mid s_{k}\right) \times \\
& p\left(z_{k}=r_{k}-c_{k} \mid s_{k}\right) \\
\alpha_{k+1}\left(s_{k+1}\right)= & \sum_{s_{k}, c_{k}} F_{k}\left(c_{k}, s_{k}, s_{k+1}\right) \\
& \times p\left(c_{k}\right) \alpha_{k}\left(s_{k}\right) \\
\beta_{k}\left(s_{k}\right)= & \sum_{s_{k+1}, c_{k}} F_{k}\left(c_{k}, s_{k}, s_{k+1}\right) \\
& \times p\left(c_{k}\right) \beta_{k+1}\left(s_{k+1}\right)
\end{aligned}
$$

and the completion stage

$$
U_{k}\left(c_{k}\right)=\sum_{s_{k}, s_{k+1}} \alpha_{k}\left(s_{k}\right) \beta_{k+1}\left(s_{k+1}\right) F_{k}\left(c_{k}, s_{k}, s_{k+1}\right)
$$

$\alpha_{k}\left(s_{k}\right)$ and $\beta_{k}\left(s_{k}\right)$ are referred to as the forward and backward filters and $F_{k}\left(c_{k}, s_{k}, s_{k+1}\right)$ represents the branch metric of the trellis diagram. We initialize the forward filter with probabilities $P_{m}$ to be in each state 
and the backward filter with 1 :

$$
\begin{aligned}
\alpha_{1}(m) & =P_{m} \quad, \quad m \in\{0,1, \ldots 6\} \\
\beta_{K+1}(m) & =1 \quad, \quad m \in\{0,1, \ldots 6\}
\end{aligned}
$$

Moreover, in our configuration of the PMC6 model,

$$
p\left(z_{k}=r_{k}-c_{k} \mid s_{k}=m\right)=\frac{1}{\sigma_{m} \sqrt{2 \pi}} e^{-\frac{\left(r_{k}-c_{k}-\mu_{m}\right)^{2}}{2 \sigma_{m}^{2}}},
$$

with $m=0,1, \ldots 6$. The probability $p\left(c_{k} \mid R_{1}^{K}\right)$ is proportional to $p\left(c_{k}\right) U_{k}\left(c_{k}\right)$ by a positive coefficient that is irrelevant for the detection process [11], [13]. From the same equation system, we can derive the probability $p\left(s_{k} \mid R_{1}^{K}\right) \propto \alpha_{k}\left(s_{k}\right) \beta_{k}\left(s_{k}\right)$ that the state $s_{k}$ is the actual channel state at the moment $k$.

Using the transition matrix of our model, the detector can calculate the metrics required for the decoding. To evaluate the relevance of our model implementation into the MAP receiver, we compare the output of the receiver for three cases: for a classic AWGN receiver, when the detector uses MC2 model and when the detector uses our proposed PMC6 model.

\section{RESULTS}

\section{A. Simulated noise}

We simulate an information transmission from a transmitter to a receiver where the channel is disturbed by an additive impulsive noise generated with our PMC6 model. We compare the received Bit-Error Rate (BER) when the receiver considers the noise being Gaussian, MC2 and PMC6. We simulate the transmission over 20 millions of samples and we use an LDPC encoder/decoder configured with the DVB-S2 standard, with $\mathrm{R}=1 / 2$ and the codeword length is 64800 bits.

The parameters of the noise in the channel are shown in Table II; the parameters of the models MC2 and PMC6, that are used in the detector block, are estimated with our impulse detection method [4] applied to the synthetic impulsive noise. We choose a high memory configuration of the model because it provides the best conditions to observe that the receiver manages to mitigate correlated impulsive noise [10]. We increase the memory of the model by representing impulses with long durations. The probability to return to the background noise $p_{10}$ in Table I characterizes the impulse duration and then the memory of the model.

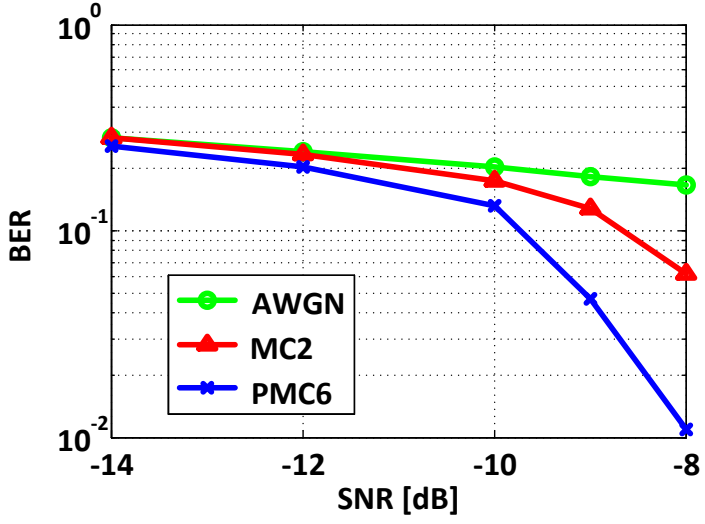

Fig. 3. BER for Optimum receiver designed with impulsive noise models; for PMC6, $p_{01}=1.271 \times 10^{-4}, p_{10}=4.689 \times 10^{-4}$ and $p_{f}=0.16$.

We observe that the receiver offers close-optimum performances for noise generated with the PMC6 model (Figure 3), since the PMC6 parameters used for the receiver are not exactly the same as the parameters used to generate the channel noise with PMC6 model. We have used Gaussian parameters estimated from our measurements (Table II); however, we have generated 10 times more impulses and for an impulse duration 10 times longer (probabilities shown in Figure 3). Hence, we observe that the more impulsive the noise, the more efficient the MAP receiver becomes with PMC6. The receiver using PMC6, starts having a lower BER at low SNR (Figure 3).

\section{B. Measured noise}

We decide now to test the MAP receiver with measured noise from an existing substation owned by HydroQuébec. We have performed many impulsive noise measurements in wide band [2], [3] and with a measurement setup that is composed of a bandpass filter and a LNA [2], we focus on the 700-800 MHz band. As we can observe in Figure 4, impulsive noise in the substation is composed of damped oscillations. We simulate the communication and add the measured noise to the received signal over 20 millions of samples. Figure 5 shows that the receiver manages to mitigate the impact of impulsive noise. Impulsive noise measured in substation is composed of shorter impulses which makes the detection of the impulses more difficult, but not impossible. We still obtain better performances than using a receiver implemented with a BernoulliGaussian model with memory. According to figures 3 and 5, smaller impulses make the detector less efficient for models with memory and the receiver starts being 
TABLE II

PARAMETERS OF THE GAUSSIAN DISTRIBUTIONS FOR PMC6 MODEL GENERATING THE CHANNEL NOISE.

\begin{tabular}{|c|c|c|c|c|c|c|c|}
\hline state $m$ & 0 & 1 & 2 & 3 & 4 & 5 & 6 \\
\hline$\mu_{m}$ & 0 & 0.0105 & 0.0475 & 0.0105 & -0.0114 & -0.0494 & -0.0114 \\
\hline$\sigma_{i}$ & 0.00433 & 0.0075 & 0.0198 & 0.0075 & 0.0079 & 0.0195 & 0.0079 \\
\hline
\end{tabular}

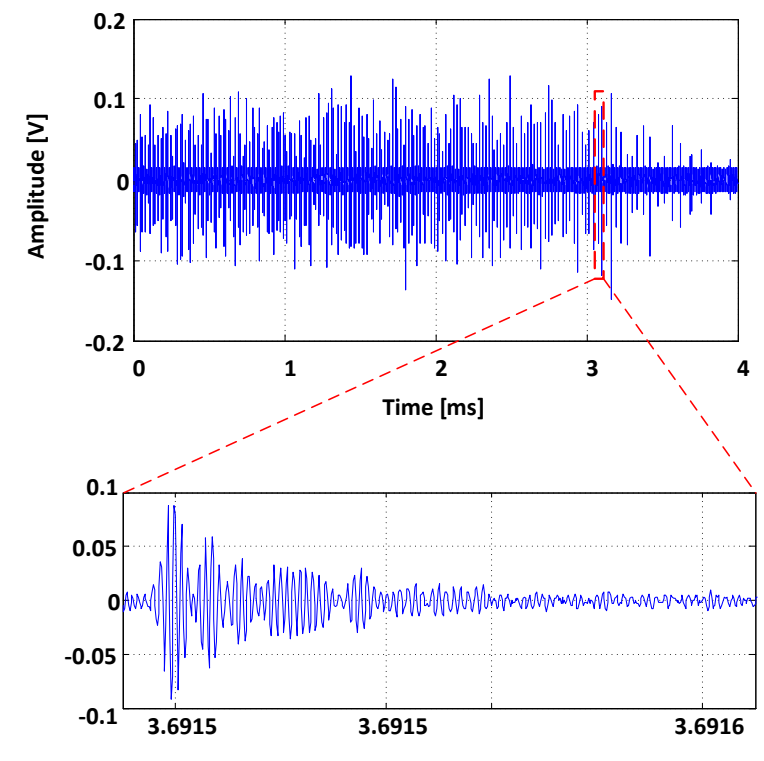

Fig. 4. Impulsive noise measured in a $735 \mathrm{kV}$ substation own by Hydro-Québec, Band: $700 \mathrm{MHz}-800 \mathrm{MHz}$, Sampling frequency = $5 \mathrm{GHz}$.

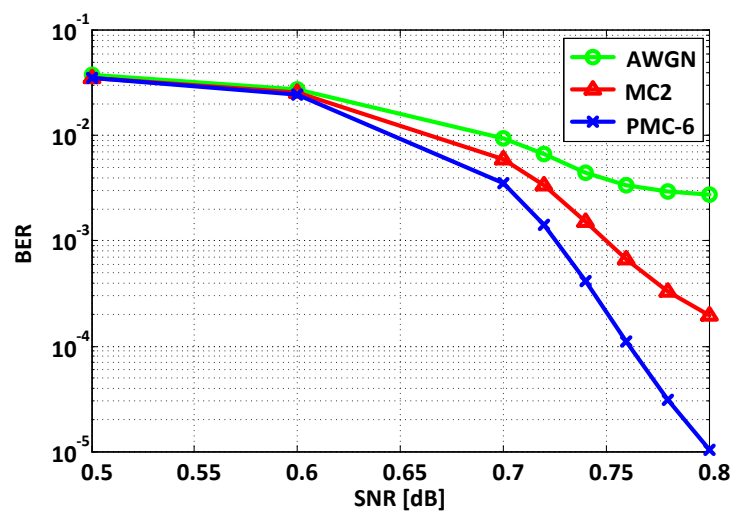

Fig. 5. BER for MAP receivers under measured impulsive noise. efficient at higher SNR than with synthetic noise. We can explain this observation by remarking that shorter impulses provide parameters that decrease the memory of the model. In [10] the authors have noticed that when the channel has more memory the receiver performs better.

\section{CONCLUSion}

A MAP receiver that uses our PMC6 model can mitigate the impact of correlated impulsive noise. Although the model is more complex in terms of configuration and parameters than with MC2 model, the receiver manages to calculate the probabilities useful to the detector and for specific scenarios of impulsive noise. The longer the impulses last and the more performing the receiver becomes when using our PMC6 model. The use of the model in the receiver will depend on the noise characteristics. We have verified that the receiver can work with different models with memory by using measured impulsive noise from an existing substation for the $700 \mathrm{MHz}-800 \mathrm{MHz}$ band. The PMC6 model can be easily used by the MAP receiver to mitigate the impact of impulsive noise in substation and the performances will increase with the impulse duration.

As future work, we recommend to study the impulsive noise characteristics to estimate the number of Markov states required to offer the best performances at the receiver. For a given sequence of noise samples, we would be able to determine the optimum model based on the duration, the rate and the variance of the impulses.

\section{ACKNOWLEDGMENT}

This work was supported by Hydro-Québec, the Natural Sciences and Engineering Research Council of Canada and McGill University in the framework of the NSERC/Hydro-Québec/McGill Industrial Research Chair in Interactive Information Infrastructure for the Power Grid.

\section{REFERENCES}

[1] C. Ozansoy, Modelling and Object Oriented Implementation of IEC 61850. Lambert Academy Publishing, 2010. 
[2] F. Sacuto, F. Labeau, J. Béland, M. Kassouf, S. Morin, S. Poirier, and B. Agba, "Impulsive Noise Measurement in Power Substations for Channel Modeling in ISM Band," in 2012 CIGRE Canada Conference, Sept 2012.

[3] F. Sacuto, F. Labeau, and B. Agba, "Evolution of the RF Characteristics of the Impulsive Noise in High Voltage Environment," in IEEE SmartGridComm 2012 Workshop, Oct 2012, pp. $686-691$.

[4] — "Wide band time-correlated model for wireless communications under impulsive noise within power substation," IEEE Transactions on Wireless Communications, vol. 13, no. 3, pp. 1449-1461, March 2014.

[5] — " "Fuzzy c-means algorithm for parameter estimation of partitioned markov chain impulsive noise model," in IEEE International Conference on Smart Grid Communications (SmartGridComm), 2013, Oct 2013, pp. 348-353.

[6] K. Saaifan and W. Henkel, "Decision boundary evaluation of optimum and suboptimum detectors in class-a interference," IEEE Transactions on Communications, vol. 61, no. 1, pp. 197205, January 2013.

[7] H. Ferreira, L. Lampe, J. Newbury, and T. Swart, Power Line Communications: Theory and Applications for Narrowband and Broadband Communications Over Power Lines. John Wiley \& Sons, 2010.
[8] M. Zimmermann and K. Dostert, "Analysis and modeling of impulsive noise in broad-band powerline communications," IEEE Transactions on Electromagnetic Compatibility, vol. 44, no. 1 , pp. $249-258$, feb 2002.

[9] A. Spaulding and D. Middleton, "Optimum reception in an impulsive interference environment-part i: Coherent detection," IEEE Transactions onCommunications, vol. 25, no. 9, pp. 910923, Sep 1977.

[10] D. Fertonani and G. Colavolpe, "On reliable communications over channels impaired by bursty impulse noise," IEEE Transactions on Communications, vol. 57, no. 7, pp. $2024-2030$, july 2009.

[11] G. Ndo, F. Labeau, and M. Kassouf, "A markov-middleton model for bursty impulsive noise: Modeling and receiver design," IEEE Transactions on Power Delivery, vol. 28, no. 4, pp. 2317-2325, Oct 2013.

[12] L. Bahl, J. Cocke, F. Jelinek, and J. Raviv, "Optimal decoding of linear codes for minimizing symbol error rate (corresp.)," IEEE Transactions on Information Theory, vol. 20, no. 2, pp. 284-287, Mar 1974.

[13] F. Kschischang, B. Frey, and H.-A. Loeliger, "Factor graphs and the sum-product algorithm," IEEE Transactions on Information Theory, vol. 47, no. 2, pp. 498-519, Feb 2001. 